Primer registro de leucismo en Glossophaga soricina valens (Phyllostomidae: Chiroptera) en Perú

\title{
First record of leucism in Glossophaga soricina valens (Phyllostomidae: Chiroptera) from Peru
}

\author{
Dennisse Ruelas ${ }^{1,2 *}$, Miluska Ramos ${ }^{1}$, Mercedes Molina ${ }^{1}$ y Víctor Pacheco ${ }^{1,3}$ \\ ${ }^{1}$ Museo de Historia Natural, Universidad Nacional Mayor de San Marcos, Av. Arenales 1256, Jesús María. Lima, Perú. Email: dennisse. \\ ruelas@unmsm.edu.pe (DR), miluskarsanchez@gmail.com (MR), mercedes.molina.veramendi@gmail.com (MM). \\ ${ }^{2}$ Museo de Historia Natural, Universidad Nacional de San Agustín, Av. Alcides Carrión s/n Facultad de Biología. Arequipa, Perú. \\ ${ }^{3}$ Instituto de Investigaciones de Ciencias Biológicas "Antonio Raimondi". Facultad de Ciencias Biológicas, Universidad Nacional \\ Mayor de San Marcos. Lima, Perú. Email: vpachecot@unmsm.edu.pe (VP) \\ ${ }^{*}$ Corresponding author
}

Leucism is a genetic condition that manifest as the total or partial lack of coloration, as result of a deficiency in the melanin deposit in the skin and fur. We present the first occurrence of leucism in Glossophaga soricina valens from Peru, a nectarivorous species of the western slope of the Andes from Peru and Ecuador, and the Marañon valley. Also, we discuss some implications of this occurrence, and update the list of bat species recorded with leucism in the America.

El leucismo es una condición genética expresada externamente como la ausencia de coloración parcial o total resultante de una deficiencia en el depósito de melanina en la piel y el pelo. Presentamos el primer caso de leucismo en Glossophaga soricina valens para Perú, especie nectarívora de la vertiente occidental de los Andes de Perú y Ecuador, también presente en la cuenca del río Marañón. Se discuten algunas implicancias sobre este hallazgo y actualizamos la lista de especies de murciélagos registrados con leucismo en América.

Key words: albinism; checklist; deficiency; inbreeding.

○ 2016 Asociación Mexicana de Mastozoología, www.mastozoologiamexicana.org

\section{Introducción}

El leucismo es una condición genética poco frecuente manifestado como la ausencia total o parcial de la coloración en la piel y el pelo como resultado de una deficiencia en el depósito de melaninas (Buckley 1982; van Grouw 2006). La causa del leucismo es atribuida principalmente a la expresión de genes mutantes que producen una migración fallida de los melanoblastos (células productoras de pigmentos) de la cresta neural a la capa basal de la epidermis durante el desarrollo embrionario, ocasionando que muy pocos o ningún melanoblasto llegue a ciertas zonas, generando así la ausencia de color en estas partes (Mayer 1973; Ericsson et al. 1992; Buckley 1982; van Grouw 2006, 2012). Las zonas afectadas por el leucismo normalmente se observan blancas o rosadas debido a que las melaninas (melanina, eumelanina y feomelanina) no pueden depositarse (Miller 2005).

Esta anomalía se ha registrado en diferentes murciélagos en América, especialmente en aquellos de la familia Phyllostomidae (Uieda 2000). En Perú son pocos los reportes de murciélagos con alguna anomalía en la pigmentación, hasta ahora el leucismo se ha registrado en seis especies: Artibeus anderseni, Chiroderma trinitatum, Desmodus rotundus, Phyllostomus hastatus, Sturnira lilium y Molossus rufus (Medina y López 2010; Diaz y Linares 2012; Tello et al. 2014). En la presente nota reportamos el primer caso de leucismo en el murciélago nectarívoro Glossophaga soricina valens, distribuido en el occidente de los Andes de Perú, y actualizamos la lista de especies de murciélagos reportadas con leucismo en América. 


\section{Material y metodos}

El registro se hace con base en un individuo macho adulto, colectado el 07 de noviembre del 2015, y depositado en el Museo de Historia Natural de la Universidad Nacional de San Marcos (MUSM) con código MUSM 44121. Junto a éste fue capturado otro individuo de la misma especie, el cual no mostraba alguna anomalía en la coloración. Los especímenes fueron capturados en redes de neblina de $12 \mathrm{~m} \times 2.5 \mathrm{~m}$ que permanecieron abiertas desde las 20:00 horas hasta las 22:30 horas, con un esfuerzo de muestreo de 5 redes-noche, como parte de un monitoreo de murciélagos en el Museo de Historia Natural.

El Museo se encuentra ubicado en el departamento y provincia de Lima, distrito de Jesús María (-12 $2^{\prime} 39^{\prime \prime} \mathrm{S},-77^{\circ} 2^{\prime} 14^{\prime \prime}$ O) en la parte céntrica de Lima y rodeado de edificaciones citadinas. El Museo cuenta con un jardín de plantas de aproximadamente $5,300 \mathrm{~m}^{2}$ con irrigación artificial, con más de 120 especies botánicas entre árboles y arbustos tanto de plantas nativas como introducidas, lo que le da una apariencia de bosque ralo con zonas arbustivas y césped (Pacheco et al. 1990).

\section{Resultados y discusión}

Glossophaga soricina es un murciélago nectarívoro ampliamente distribuido en el Neotrópico (Miller 1913), contiene cinco subespecies de las cuales dos se han registrado en Perú, G. s. soricina al oriente de los Andes y a G. s. valens al occidente y en el Valle Seco del Marañón (Miller 1913; Webster 1993; Hoffmann y Baker 2001). Glossophaga soricina valens es tratada aquí como subespecie y no como una especie plena como lo sugerido por Pari et al. (2015), ya que aún no hay una revisión minuciosa del taxón que la compare con las otras subespecies y que enmiende la diagnosis de G. soricina. Revisiones conocidas han optado por mantener ese estatus (Webster 1993; Hoffmann y Baker 2001) que también es seguido aquí.

El primer caso de $G$. soricina reportado con desórdenes de pigmentación (albinismo total) carece de localidad de registro (Schneider 1925). Posteriormente en México se reportó un espécimen hembra de G. s. handleyi con albinismo parcial (García-Morales et al. 2010), luego Zalapa et al. (2014) reportaron un caso de leucismo no indicando la procedencia del espécimen. Nuestro registro corresponde al primer caso de leucismo en Glossophaga soricina valens y al primer caso de leucismo en un murcielago nectarivoro en Perú.

Consideramos que nuestro ejemplar presenta leucismo del tipo parcial (sensu Miller 2005, van Grouw 2012), ya que presenta desordenes de pigmentación en la mitad del dactilopatagio derecho y en los pelos del dedo I derecho (Figura 2A). La ausencia de la pigmentación es tal que se muestra transparente, incluso pudiéndose leer un texto a través del éste, mientras que los huesos muestran una tonalidad rosada debido a su irrigación sanguínea. El dactilopatagio izquierdo se muestra más claro en la parte distal y más oscuro cerca al cuerpo y presenta pequeñas manchas blancas dispersas (Figura 2B). El pelaje de la región dorsal del cuello y sobre las orejas presenta las puntas ( $1 / 3$ terminal) con una coloración normal, mientras que la parte basal (2/3 basal) es blanquecina. No notamos anomalías en otras partes del cuerpo o a nivel craneal. La coloración del cuerpo en general no presentó mayor alteración en la coloración y los ojos tenían una coloración y apariencia normales.

La coloración de los ojos no se ve afectada en el leucismo porque los cromatóforos del iris tienen un origen embrionario distinto a los de la piel, ya que se forman a partir del tubo neural, mientras que los de piel, a partir de la cresta neural (Solari 2004). Contrariamente, en el albinismo la coloración de los ojos está ausente y se aprecian de color rojo debido a la 
Figura 1. Leucismo en Glossophaga soricina valens (MUSM 44121): A) Dactilopatagio derecho y B) dactilopatagio izquierdo.

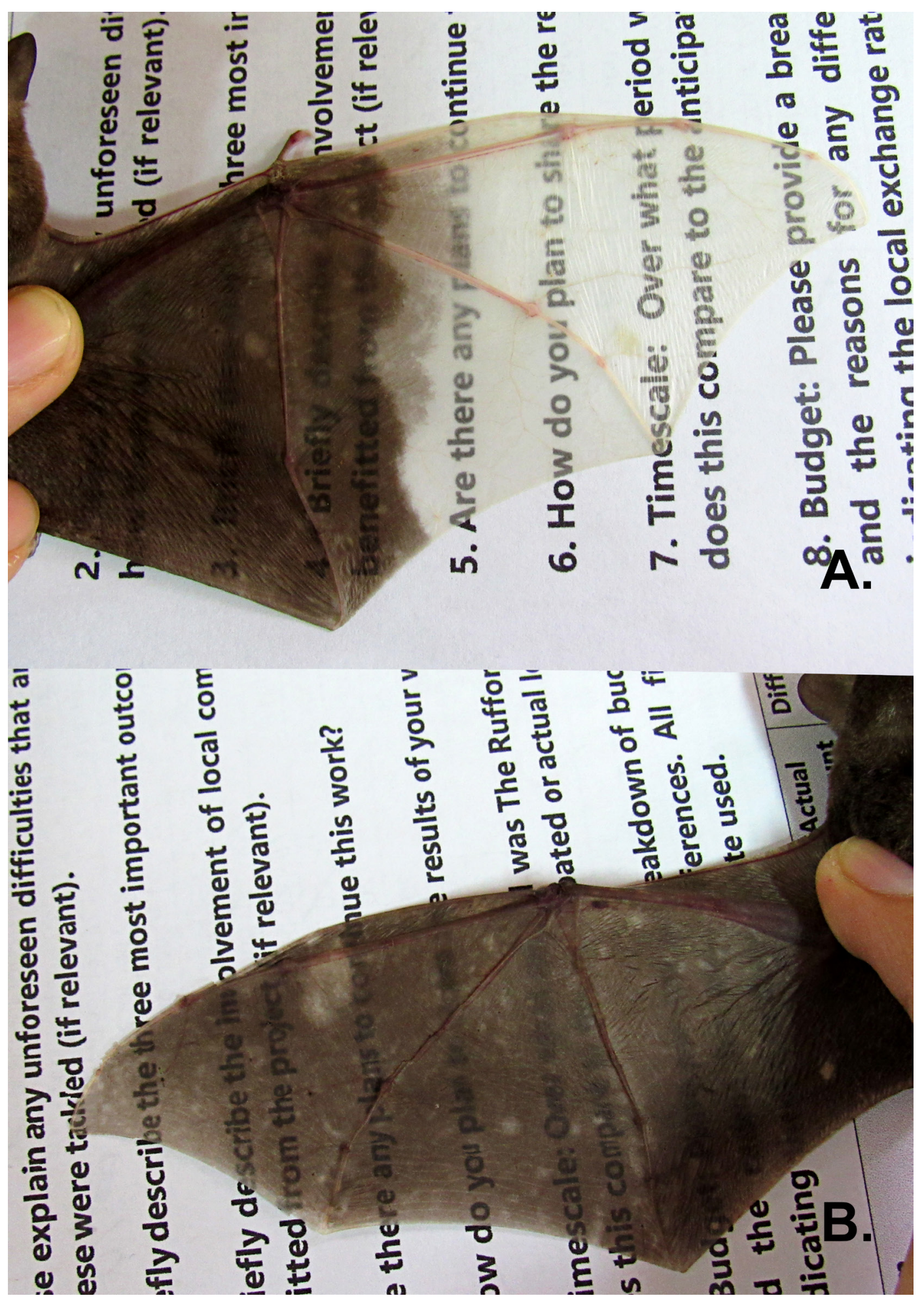

reflexión de los capilares sanguíneos de la retina (Griffiths et al. 2000) y además las partes desnudas suelen ser rosadas o rojizas (Miller 2005; van Grouw 2006).

La ausencia de coloración en individuos leucísticos no está relacionado con la ausencia o defectos en la tirosinasa, enzima que cataliza la producción de melanina (van Grouw 2012), como lo sugirieron Sage (1962) y Krecsák (2008), ya que esta enzima se encontraría presente en éstos y 
la producción de melaninas sería normal (Urcola 2011), contrariamente en individuos albinos esta enzima se presenta defectuosa (Armstrong y Bennett 1982).

Sin embargo, el leucismo en murciélagos ha sido tratado incorrectamente como albinismo parcial por varios autores (Metzger 1956; Feng et al. 2007; Hernández-Mijangos 2009; Boada y Tirira 2010; MarinVásquez et al. 2010; Acosta et al. 2011; Mantilla-Meluk y Jiménez-Ortega 2011; Díaz y Linares 2012) han reportado especies con ausencia de coloración total o parcial en la piel y el pelo, pero con la coloración normal de los ojos.

A la fecha, en América se han reportado 45 especies de murciélagos con leucismo (Tabla 1), la familia con más casos es Phyllostomidae ( $n=25,55.5 \%$ ), seguida de Vespertilionidae ( $n=10,22.2 \%$ ), Molossidae ( $n=9,20.0 \%)$ y Mormoopidae $(n=1,2.2 \%)$. Las especies nectarívoras tienen menor cantidad de casos con tres especies, mientras que los frugívoros (16 especies) e insectívoros (21 especies) presentan mayor cantidad de casos. Los países con más especies reportadas con esta anomalía son México $(n=13)$,

Tabla 1. Especies de murciélagos registrados con leucismo en países de América ( ${ }^{*}=$ Sin ejemplares de referencia).

\begin{tabular}{|c|c|c|}
\hline ESPECIES & PAÍS & REFERENCIA \\
\hline \multicolumn{3}{|l|}{ FAMILIA PHYLLOSTOMIDAE } \\
\hline Anoura caudifer & Colombia, Venezuela & Linares (1967), Velandia-Perilla et al. (2013). \\
\hline Artibeus amplus & Colombia, Venezuela & Marin-Vasquez et al. (2013), Muñoz-Romo et al. (2015) \\
\hline Artibeus anderseni & Perú & Tello et al. (2014) \\
\hline Artibeus jamaicensis & México, Colombia & $\begin{array}{l}\text { Hernández-Mijangos (2009), Sánchez-Hernández et al. (2010), } \\
\text { Marin-Vasquez et al. (2010), García-Morales et al. (2012, 2010), } \\
\text { Sánchez-Hernández et al. (2012) }\end{array}$ \\
\hline Artibeus lituratus & $\begin{array}{l}\text { México, Costa Rica, } \\
\text { Colombia, Brasil }\end{array}$ & $\begin{array}{l}\text { Sánchez et al. (1989), López-Wilchis et al. (2012), Sánchez- } \\
\text { Hernández et al. (2012), García-Morales et al. (2013), Souza et } \\
\text { al. (2013), Velandia-Perilla et al. (2013), Olarte-González et al. } \\
\text { (2014), Zalapa et al. (2014)* }\end{array}$ \\
\hline Artibeus phaeotis & México, Guatemala & Sánchez et al. (1989), Trujillo y Barahona (2014) \\
\hline Artibeus planirostris & Colombia, Brasil & Marin-Vasquez et al. (2013), Guimaraes et al. (2014)*, Chacón et al. (2015) \\
\hline Artibeus watsoni & México & Hernández-Mijangos (2009) \\
\hline Carollia brevicauda & Colombia, Venezuela & $\begin{array}{l}\text { Soriano et al. (1993), Marin-Vasquez et al. (2010), Velandia- } \\
\text { Perilla et al. (2013) }\end{array}$ \\
\hline Carollia perspicillata & $\begin{array}{l}\text { Trinidad y Tobago, } \\
\text { Ecuador, Brasil }\end{array}$ & $\begin{array}{l}\text { Goodwin y Greenhall (1961), Boada y Tirira (2010), Rocha et al. } \\
\text { (2013) }\end{array}$ \\
\hline Carollia sowelli & México & Hernández-Mijangos (2009) \\
\hline Chiroderma trinitatum & Perú & Tello et al. (2014) \\
\hline Dermanura rosenbergi & Colombia & Velandia-Perilla et al. (2013) \\
\hline Desmodus rotundus & Perú, & Tello et al. (2014) \\
\hline Glossophaga soricina & ¿?, México, Perú & $\begin{array}{l}\text { Schneider (1925), García-Morales et al. (2010), Zalapa et al. } \\
(2014)^{*} \text {, este trabajo }\end{array}$ \\
\hline Lonchophylla robusta & Colombia & Velandia-Perilla et al. (2013) \\
\hline Lophostoma silvicolum & Colombia & Marin-Vasquez et al. (2010) \\
\hline Macrotus waterhousii & México & Sánchez et al. (1989), Sánchez-Hernandez et al. (2012) \\
\hline Micronycteris microtis & Bolivia & Acosta et al. (2011) \\
\hline Phyllostomus discolor & Colombia, Brasil & Treitler et al. (2013), Marin-Vasquez et al. (2013) \\
\hline Phyllostomus hastatus & Perú & Díaz y Linares (2012) \\
\hline Sturnira erythromos & Colombia, Argentina & Barquez et al. (2003)*, Roncancio y Ramírez-Chaves (2008) \\
\hline Sturnira lilium & México, Brasil, Perú & $\begin{array}{l}\text { Medina y López (2010), Kaku-Oliveira et al. (2014)*, Zalapa et al. } \\
(2014)^{*}\end{array}$ \\
\hline Sturnira ludovici & México & García-Morales et al. (2012), Sánchez-Hernández et al. (2012) \\
\hline Uroderma bilobatum & Colombia & Mantilla-Meluk y Jiménez-Ortega (2011) \\
\hline
\end{tabular}




\begin{tabular}{|c|c|c|}
\hline Pteronotus cuadridens & Puerto Rico & Rodríguez-Durán y Kunz (1992) \\
\hline \multicolumn{3}{|l|}{ FAMILIA MOLOSSIDAE } \\
\hline Eumops bonaeriensis & Argentina & Barquez et al. (2003)* \\
\hline Eumops glaucinus & Argentina & Barquez et al. (2003)* \\
\hline Eumops patagonicus & Argentina & Barquez et al. (2003)* \\
\hline Molossus rufus & Perú & Díaz y Linares (2012) \\
\hline Molossus sinaloae & Costa Rica & Zalapa et al. (2014)* \\
\hline Nyctinomops femorosaccus & México & Zalapa et al. (2014)* \\
\hline Nyctinomops laticaudatus & Brasil & Geiger y Pacheco (2006) \\
\hline Tadarida brasiliensis & $\begin{array}{l}\text { EE. UU., México, } \\
\text { Argentina }\end{array}$ & $\begin{array}{l}\text { Glass (1954), Herreid y Davis (1960), Caire y Thies (1988), Barquez et } \\
\text { al. (2003)*, Sánchez-Hernández et al. (2012), Romano et al. (2015) }\end{array}$ \\
\hline Tadarida femorosacca & EE. UU. & Mitchell (1963) \\
\hline \multicolumn{3}{|l|}{ FAMILIA VESPERTILIONIDAE } \\
\hline Eptesicus furinalis & Argentina & Barquez et al. (2003)*, Idoeta et al. (2011) \\
\hline Eptesicus fuscus & EE. UU. & Trapido y Crowe (1942) \\
\hline Myotis grisescens & EE. UU. & Tuttle (1961) \\
\hline Myotis levis & Brasil & Miranda et al. (2010) \\
\hline Myotis lucifugus & Canadá, EE. UU. & Trapido y Crowe (1942), Walley (1971), Talerico et al. (2008) \\
\hline Myotis riparius & Argentina* & Barquez et al. $(2003)^{*}$ \\
\hline Myotis sodalis & EE. UU., México & Metzger (1956), Barbour y Davis (1970), Caire y Thies (1988) \\
\hline Myotis velifer & EE. UU., México & Rogers (1965) \\
\hline Nycticeius humeralis & EE. UU. & Easterla y Watkins (1968) \\
\hline Perimyotis subflavus & EE. UU. & Trapido y Crowe (1942), Goslin (1947), Blair (1948) \\
\hline
\end{tabular}

Colombia $(n=12)$ y EE. UU. $(n=9)$. La mayoría de los registros han sido publicados a partir del año 2000.

La importancia biológica de este patrón atípico aún es desconocida pero se ha sugerido que podría reducir la eficacia biológica o "fitness" de los individuos afectados (Bensch et al. 2000), además de hacerlos más visibles ante los depredadores y menos atractivos durante la temporada de reproducción (Rees 2004; Torres y Franke 2008; Jogahara et al. 2008).

Normalmente la expresión de los genes mutantes se da en baja frecuencia; sin embargo, la endogamia aumenta la probabilidad de que éstos se expresen, por lo que el leucismo se podría presentar con mayor frecuencia en poblaciones pequeñas y aisladas (Glass 1954; Bensch et al. 2000; Chẹtnicki et al. 2007). Por lo que este tipo de anomalías podría mostrarnos una evidencia indirecta de la variabilidad genética subyacente de las poblaciones que lo portan (Jehl 1985). Por otro lado, para Uieda (2000) los murciélagos con refugios cerrados como minas, cuevas, edificios, galerías y viviendas tienen mayor frecuencia de presentar algún tipo de desorden en la coloración que aquellos que habitan en refugios abiertos.

García-Morales et al. (2013) observaron que el feto de una hembra leucística de Artibeus lituratus no presentaba anomalías en la coloración, atribuible a la condición recesiva del leucismo; sin embargo, la cría no dejaría de ser portadora de este gen mutante. Este hallazgo sugiere que hembras leucísticas podrían estar reproduciéndose en ambientes naturales, por lo que el atractivo en los murciélagos no sería morfológico sino comportamental u hormonal. Esto sustentaría lo documentado por otros autores que indicaron que individuos leucísticos pueden 
coexistir con normalidad con sus conespecíficos por largos periodos de tiempo en sus refugios naturales (López-Wilchis y León 2012; Sánchez-Hernández et al. 2010).

Glossophaga soricina valens es uno de los murciélagos mejor adaptados a las ciudades y estaría dispersándose con normalidad, habitando en refugios cerrados (casas, iglesias) y abiertos (parques), con poblaciones aún desconocidas en estructura y cantidad, por lo que no podemos hacer inferencias sobre algún proceso de endogamia o reducción de la eficacia biológica por el tipo de refugio o estado poblacional que tienen. Por lo expuesto, es necesario realizar monitoreos constantes para detectar a las poblaciones afectadas, observar el comportamiento de los individuos leucísticos dentro de su colonia y realizar estudios en genética de poblaciones en las especies citadinas, además también se necesitan más estudios para conocer y entender todas las causas y consecuencias de estas anomalías en la coloración de los murciélagos.

\section{Agradecimientos}

Agradecemos muy cordialmente a O. Ruelas del Museo de Historia Natural de la Universidad Nacional de San Agustín (MUSA) por su apoyo y comentarios en la redacción , y también a los revisores que ayudaron a mejorar de la presente nota.

\section{Literatura citada}

Acosta, L. H., M. Saldías, y L. A. Nuñez. 2011. Historia natural del murciélago de hojas largas (Micronycteris microtis, Miller 1898), en la serranía de Incahuasi, Santa CruzBolivia. Kempffiana 7:19-33.

Armstrong, F. B., y T. P. Bennett. 1982. Bioquímica. Editorial Reverté S. A. Barcelona, España.

Barbour, R. W., y W. H. Davis. 1969. Bats of America. University Press of Kentucky. Lexington, EE. UU.

Barquez, R. M., L. V. Carrizo, L. I. Ferro, D. A. Flores, M. I. Mollerach, M. S. Sánchez, y A. P. García López. 2003. Primer caso de albinismo total para Sturnira erythromos (Tschudi, 1844) - (Chiroptera - Phyllostomidae). Chiroptera Neotropical 9:166-169.

Bensch, S., B. Hansson, D. Hasselquist, y B. Nielsen. 2000. Partial albinism in a semi-isolated population of great reed warblers. Hereditas 133:167-170.

BLAIR, W. F. 1948. A color pattern aberration in Pipistrellus subflavus subflavus. Journal of Mammalogy 29:178-179.

Boada, C., y D. G. TiriRa. 2010. First record of partial albinism (leucism) in Carollia perspicillata (Phyllostomidae) in Ecuador. Chiroptera Neotropical 16:755-757.

Buckley, P. A. 1982. Avian genetics. Pp. 21-110 en Diseases of cage and aviary birds (Petrak M. L., ed.). Lea and Febiger. Philadelphia, EE. UU.

CAIRE, W., Y M. Thies. 1988. Notes on the occurrence of morphological and color aberrations in bats from Oklahoma, Missouri, and Mexico. Proceedings of the Oklahoma Academy of Science 68:75-76.

Chacón, J. J., C. M. González, y J. Ballesteros. 2015. Records of leucism in Artibeus planirostris (Chiroptera: Phyllostomidae) in Colombia. Acta Zoológica Mexicana (n. s.) 31:125-128.

Chętnicki, W., S. Fedyk, y U. Bajkowska. 2007. Cases of coat colour anomalies in the common shrew, Sorex araneus L. Folia biológica (Kraków) 55:73-76.

Díaz, M. M., y V. H. Linares. 2012. Refugios naturales y artificiales de Murciélagos (Mammalia: Chiroptera) en la selva baja en el noroeste de Perú. Gayana 76:117-130. 
Easterla, D. A., y L. C. Watkins. 1968. An aberrant evening bat. Southwestern Naturalist 13:447-448.

Erickson, C. A., T. D. Duong, y W. Tosney. 1992. Descriptive and experimental analysis of the dispersion of neural crest cells along the dorsolateral path and their entry into ectoderm in the chick embryo. Developmental biology 151:251-272.

FENG, L., T. JIA, Z. SHu-YI, Y C. QI-CAI. 2007. A partial albino bat of Miniopterus magnate found in Anhui, China. Zoological Research 28:443-445.

García-Morales, R., A. E. Rojas-Martínez, E. S. Ávila Gómez, y C. E. Moreno. 2013. Leucism in the giant fruit-eating bat (Artibeus lituratus Olfers, 1818) in the state of Hidalgo, Mexico. Chiroptera Neotropical 19:1212-1215.

García-Morales, R., D. Tejada Duran, E. S. Ávila Gómez, C. E. Moreno, y M. S. Akmentins. 2012. Registro de leucismo en Sturnira ludovici y Artibeus jamaicensis (Phyllostomidae) en México. Chiroptera Neotropical 18:1101-1105.

García-Morales, R., E. G. Gordillo-Chávez, y J. Bello-Gutiérrez. 2010. Primer registro de albinismo en Glossophaga soricina (Phyllostomidae) en México. Chiroptera Neotropical 16:743-747.

Geiger, D., y S. M. Pacheco. 2006. Registro de albinismo parcial em Nyctinomops laticaudatus (E. Geoffroy, 1805) (Chiroptera: Molossidae) no sul do Brasil. Chiroptera Neotropical 12:250-254.

GLass, B. P. 1954. Aberrant coloration in Tadarida mexicana. American Midland Naturalist 52:400-402.

Griffiths, A. J. F., W. M. Gelbart, J. H. Miller, y R. L. White. 2000. Genética moderna. McGrawHill-Interamericana. Madrid, España.

Goodwin, G. G., y A. M. Greenhall. 1961. A review of the bats of Trinidad and Tobago: descriptions, rabies infection, and ecology. American Museum of Natural History. 122:1-271.

Gosuin, R. 1947. A bat with white wing tips. Journal of Mammalogy 28:62.

Guimaraes, M., T. Midori, N. Kaku-Oliveira, y W. Uieda. 2014. Primer registro de leucismo en Artibeus planirostris (Spix, 1823) (Phyllostomidae). Pp 208 en Libro de resúmenes del I Congreso latinoamericano y del Caribe de murciélagos. Pontificia Universidad Católica del Ecuador. Quito, Ecuador.

Hernández-Mijangos, L. A. 2009. Registros de albinismo parcial en tres especies de filostómidos (Chiroptera: Phyllostomidae) en Chiapas, México. Chiroptera Neotropical 15:441-445.

Herreid II, C. F., y R. B. Davis. 1960. Frequency and placement of white fur on Free-tailed bats. Journal of Mammalogy 41:117-119.

Hoffmann, F. G., Y R. J. Baker. 2001. Systematics of bats of the genus Glossophaga (Chiroptera: Phyllostomidae) and phylogeography in G. soricina based on the cytochrome-b gene. Journal of Mammalogy 82:1092-1101.

Idoeta, F. M., De Santis L. J. M., y R. M. Barquez. 2011. Leucismo en Eptesicus furinalis (d'Orbigny y Gervais, 1847) (Chiroptera:Vespertilionidae) en la provincia de Corrientes, Argentina. Chiroptera Neotropical 17:985-988.

JehL, J. R. 1985. Leucism in pared grebes in western North America. The Condor 87:439-441

Jogahara, T., G. Ogura, G. Higa, O. Ishibashi, y S. Oda. 2008. Survey and capture of albinolike house mus shrews (Suncus murinus) in Okinawa, Japan, and a preliminary report regarding inheritance of the albino-like mutation. Mammal Study 33:121-124.

Kaku-Oliveira, N., J. Rezini, B. Cavalcanti, A. Costa, y F. Goss. 2014. Leucismo en Sturnira lilium (E. Geoffroy, 1810) (Phyllostomidae: Stenodermatinae) en el estado de Sao 
Paulo, Brasil. Pp. 204 en Libro de resúmenes del I Congreso latinoamericano y del Caribe de murciélagos. Pontificia Universidad Católica del Ecuador. Quito, Ecuador.

Krecsák, L. 2008. Albinism and leucism among European viperinae: a review. Russian journal of Herpetology 15:97-102.

LinARES, O. J. 1967. Albinism in the long-tongued bat, Anoura caudifera. Journal of Mammalogy 48:464-465.

López-Wilchis, R., y M. A. León. 2012. A noteworthy case of leucism in Artibeus lituratus (Chiroptera: Phyllostomidae) from Oaxaca, Mexico. Chiroptera Neotropical 18:11111114.

Mantilla-Meluk, H., y A. Jiménez-Ortega. 2011. First case of albinism in Uroderma bilobatum and its implications in the evolution of coat color patterns among Vampyressine bats. Investigación, Biodiversidad y Desarrollo 30:97-100.

Marin-Vasquez, A., I. González-Ibarra, G. Gualy, y J. D. Díaz. 2013. Aberrations in coat color pattern of Colombian bats: Isabellinae, leucism and melanocytes nevus. Online Journal of BioSciences and Informatics 4:253-265.

Marin-Vasquez, A., M. Ortega-Rincón, y H. E. Ramírez-Chaves. 2010. Records of leucism in three species of Colombian bats: Carollia brevicaudata, Artibeus jamaicensis and Lophostoma silvicolum (Phyllostomidae). Chiroptera Neotropical 16:706-709.

MAYeR, T. C. 1972. The migratory pathway of neural crest cells into the skin of mouse embryos. Developmental Biology 34:39-46.

Medina, C., y E. Lopez. 2010. Primer registro de leucismo en Sturnira lillium (Pyllostomidae) en el sureste de Perú. Chiroptera Neotropical 16:758-761.

Metzger, B. 1956. Partial albinism in Myotis sodalis. Journal of Mammalogy 37:546.

Miller, G., JR. 1913. Revision of the bats of the genus Glossophaga. Proceedings of the United States National Museum 46:413-429.

Miller, J. D. 2005. All about albinism. Missouri Conservationist 66:5-7.

Miranda, J. M. D., N. Y. Kaku-Oliveira, L. C. Munster, I. P. Bernardi, R. F. Moro-Rios, y F. C. Passos. 2010. Primeiros dados de uma colônia reprodutiva de Myotis levis (I. Geoffroy, 1824) nos campos de Palmas, Paraná, Brasil (Vespertilionidae). Chiroptera Neotropical 16:762-768.

Mitchell, H. A. 1963. Aberrant white fur in the pocketed freetailed bat. Journal of Mammalogy 44:422.

Muñoz-Romo, M., D. Ruiz, y P. Ramoni-Perazzi. 2015. First record of leucism in the giant fruiteating bat, Artibeus amplus (Chiroptera: Phyllostomidae). Chiroptera Neotropical 20:1301-1304.

Olarte-González, G., I. M. Vela-Vargas, P. Camargo, T. Jhonson, y A. Jiménez. 2014. Primer Reporte de leucismo en Artibeus lituratus (Olfers, 1818) (Phyllostomidae: Stenodermatinae) en el Caribe colombiano. Mammalogy Notes 1:7-8.

Pacheco, V., B. Paredes, J. Crosby, J. Gayoso, F. Cardoso, y H. Sisniegas. 1990. Notas sobre la avifauna en ambientes urbanos y sugerencias sobre planeamiento de parques. Publicaciones del Museo de Historia Natural UNMSM, serie A 35:1-7.

Pari, A., K. Pino, C. Medina, E. López, y H. Zeballos. 2015. Murciélagos de Arequipa, historia natural y conservación. Arequipa, Perú.

ReEs, P. 2004. Are white lion ambassadors or conservation white elephants? International Zoo News 51:484-489.

Rocha, P. A., J. A. Feijó, C. R. Donato, y S. F. Ferrari. 2013. Leucism in Seba's short-tailed bat, Carollia perspicillata (Linnaeus, 1758), from a rock shelter in northeastern Brazil. Chiroptera Neotropical 19:1151-1153. 
Rodríguez-Durán, A., Y T. H. Kunz. 1992. Pteronotus quadridens. Mammalian species 395:1-4. RoGERS, G. C. 1965. Aberrant coloration in Myotis velifer incautus. Southwestern Naturalist 10:311.

Romano, M., M. Montanı, C. Cordinı, y S. Aull. 2015. First record of Albinism in Tadarida brasiliensis (Chiroptera: Molossidae) in South America and new records of leucism in central Argentina. Chiroptera Neotropical 21:1312-1319.

Roncancio, N., y H. E. Ramírez-Chaves. 2008. Registro de leucismo en Sturnira erythromos en los Andes Centrales de Colombia. Chiroptera Neotropical 14:412-414.

SAGE, B. 1962. Albinism and melanism in birds. British Birds 55:55-201.

Sánchez, H. C., C. W. López-Forment, y H. M. A. Gurrola. 1989. Unusual coloration in three Mexican bats. Bat Research News 30:54-55.

Sánchez-Hernández, C., A. Rojas-Martínez, J. C. López-Vidal, C. Elizalde-Arellano, M. RomeroAlmaraz, M. Aguilar-López, y A. Taboada-Salgado. 2012. Leucism in five species of bats from Mexico. Chiroptera Neotropical 18:1123-1127.

Sánchez-Hernández, C., M.L. Romero-Almaraz, A. Taboada-Salgado, J. Almazán-Catalán A., G. D. SchnelL, y L. SÁnchez-VÁzquez. 2010. Five albino bats from Guerrero and Colima, Mexico. Chiroptera Neotropical 16:541-545.

SCHneider, G. 1925. Ein interessanter fall von albinismus bei Glossophaga soricina Pall. Ad. Revue Suisse de Zoologie 32:85.

Soları, A. J. 2004. Genética humana: fundamentos y aplicaciones en medicina. Editorial Médica Panamericana. Buenos Aires, Argentina.

Soriano, P., A. Utrera, y M. Sosa. 1993. Dos registros de murciélagos albinos para Venezuela. Biollania 9:149-150.

Souza, R., R. Morim, S. Felix, C. Sauwen, G. Jacob, R. Tadeu, y L. dos Santos. 2013. First record of leucism in Artibeus lituratus (Olfers, 1818) (Phyllostomidae) in Brazil. Chiroptera Neotropical 19:1216-1219.

Talerico, J. M., T. S. Jung, R. M. R. Barclay, y K. S. Melton. 2008. Aberrant coloration in a Little Brown Bat (Myotis lucifugus) from the Yukon. Northwestern Naturalist 89:198-200.

Tello, C., D. G. Streicker, J. Gomez, y P. M. Velazco. 2014. New records of pigmentation disorders in molossid and phyllostomid (Chiroptera) bats from Peru. Mammalia 78:191-197.

Torres, M., y I. Franke. 2008. Reporte de albinismo en Podiceps major, Pelecanus thagus y Cinclodes fuscus. Revista Peruana de Biología 15:105-108.

Trapido, H., Y P. E. CROWE. 1942. Color abnormalities in three genera of northeastern cave bats. Journal of Mammalogy 23:303-305.

Treitler, J. T., A. López, S. Gomes, J. F. Tenaçol JR., y R. Rocha. 2013. First record of a leucistic piebald Phyllostomus discolor (Chiroptera: Phyllostomidae). Chiroptera Neotropical 19:1179-1181.

Trujillo, L., y R. Barahona. 2014. First record of leucism in Artibeus phaeotis (Miller, 1902) (Chiroptera: Phyllostomidae) from Guatemala. Chiroptera Neotropical 20:1252-1254.

TuttLE, M. D. 1961. Notes and correspondence. Bat Banding News 2:16.

UIEDA, W. 2000. A review of complete albinism in bats with five new cases from Brazil. Acta Chiropterologica 2:97-105.

URColA, M. R. 2011. Aberraciones cromáticas en aves de la colección ornitológica del Museo Argentino de Ciencias Naturales "Bernardino Rivadavia". Revista del Museo Argentino de Ciencias Naturales 13:221-228.

VAN Grouw, H. 2006. Not every white bird is an albino: sense and nonsense about color aberrations in birds. Dutch Birding 28:79-89.

Van Grouw, H. 2012. What colour is that sparrow? A case study: colour aberrations in the house sparrow Passer domesticus. International Studies on Sparrows 36:30-55. 
Velandia-Perilla, J. H., A. P. Yusti-Muñoz, M. A. Sánchez-Martínez, y A. Giraldo. 2013. Leucismo en murciélagos de hoja nasal (Chiroptera, Phyllostomidae) de Colombia. Boletín científico del centro de museos de la Universidad de Caldas 17:87-94.

Walley, H. D. 1974. Albino little brown bat, Myotis lucifugus lucifugus, new record from Wisconsin with remarks on other abberrant bats. Canadian Field-Naturalist 88:80-81.

Walley, H. D. 1971. A leucistic little brown bat (Myotis l. lucifugus). Transactions of the Illinois Academy of Science 64:196-197.

Webster, W. D. 1993. Systematics and evolution of bats of the genus Glossophaga. Special Publications, The Museum, Texas Tech University 36:1-184.

Zalapa, S., S. Guerrero, M. Romero, y Sánchez C. 2014. Coloración atípica en murciélagos: Análisis sobre su frecuencia en América y nuevos casos para México y Costa Rica. Pp. 205 en Libro de resúmenes del I Congreso latinoamericano y del Caribe de murciélagos. Quito, Ecuador.

Submited: February 24, 2016

Reviewed: April 22, 2016

Accepted: May 19, 2016

Associated editor: Lia Méndez 